\title{
Tuning and Robustness Analysis for the Orion Absolute Navigation System
}

\author{
Greg N. Holt* \\ NASA Johnson Space Center, 2101 NASA Parkway, Houston, TX 77058 \\ Renato Zanetti ${ }^{\dagger}$ \\ Charles Stark Draper Laboratory, Houston, TX \\ Christopher D'Souza ${ }^{\ddagger}$ \\ NASA Johnson Space Center, 2101 NASA Parkway, Houston, TX 77058
}

The Orion Multi-Purpose Crew Vehicle (MPCV) is currently under development as NASA's next-generation spacecraft for exploration missions beyond Low Earth Orbit. The MPCV is set to perform an orbital test flight, termed Exploration Flight Test 1 (EFT-1), some time in late 2014. The navigation system for the Orion spacecraft is being designed in a Multi-Organizational Design Environment (MODE) team including contractor and NASA personnel. The system uses an Extended Kalman Filter to process measurements and determine the state. The design of the navigation system has undergone several iterations and modifications since its inception, and continues as a work-in-progress. This paper seeks to show the efforts made to-date in tuning the filter for the EFT-1 mission and instilling appropriate robustness into the system to meet the requirements of manned spaceflight.

Filter performance is affected by many factors: data rates, sensor measurement errors, tuning, and others. This paper focuses mainly on the error characterization and tuning portion. Traditional efforts at tuning a navigation filter have centered around the observation/measurement noise and Gaussian process noise of the Extended Kalman Filter. While the Orion MODE team must certainly address those factors, the team is also looking at residual edit thresholds and measurement underweighting as tuning tools. Tuning analysis is presented with open loop Monte-Carlo simulation results showing statistical errors bounded by the 3-sigma filter uncertainty covariance.

The Orion filter design uses 24 Exponentially Correlated Random Variable (ECRV) parameters to estimate the accel/gyro misalignment and nonorthogonality. By design, the time constant and noise terms of these ECRV parameters were set to manufacturer specifications and not used as tuning parameters. They are included in the filter as a more analytically correct method of modeling uncertainties than ad-hoc tuning of the process noise. Tuning is explored for the powered-flight ascent phase, where measurements are scarce and unmodelled vehicle accelerations dominate. On orbit, there are important trade-off cases between process and measurement noise. On entry, there are considerations about trading performance accuracy for robustness. Process Noise is divided into powered flight and coasting flight and can be adjusted for each phase and mode of the Orion EFT-1 mission. Measurement noise is used for the integrated velocity measurements during pad alignment. It is also used for Global Positioning System (GPS) pseudorange and deltarange measurements during the rest of the flight.

The robustness effort has been focused on maintaining filter convergence and performance in the presence of unmodeled error sources. These include unmodeled forces on the vehicle and uncorrected errors on the sensor measurements. Orion uses a single-frequency, non-keyed GPS receiver, so the effects due to signal distortion in Earth's ionosphere and troposphere are present in the raw measurements. Results are presented showing the efforts

*Navigation Engineer, Flight Dynamics Division/Mission Operations, AIAA Senior Member

${ }^{\dagger}$ Senior Engineer, AIAA Senior Member

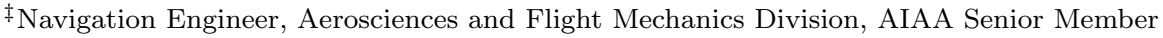


to compensate for these errors as well as characterize the residual effect for measurement noise tuning. Another robustness tool in use is tuning the residual edit thresholds. The trade-off between noise tuning and edit thresholds is explored in the context of robustness to errors in dynamics models and sensor measurements. Measurement underweighting is also presented as a method of additional robustness when processing highly accurate measurements in the presence of large filter uncertainties. 